\title{
Mortalidade Hospitalar na Doença de Parkinson: Análise Retrospetiva num Hospital Terciário Português
}

\section{Hospital Mortality in Parkinson's Disease: Retrospective Analysis in a Portuguese Tertiary Centre}

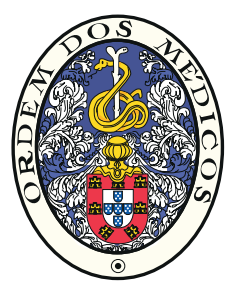

\author{
Joana MARTINS $\square^{1}$, Adriana RUA ${ }^{1}$, Nuno Vila CHÃ 1 \\ Acta Med Port 2016 May;29(5):315-318 - http://dx.doi.org/10.20344/amp.7070
}

RESUMO

Introdução: A doença de Parkinson está associada a elevada mortalidade hospitalar. O sexo masculino, o início tardio da doença, o grau de incapacidade e a coexistência de deterioração cognitiva ou de depressão têm sido apontados como fatores de risco. A pneumonia e as doenças cardiovasculares são as principais causas de morte.

Objetivo: Explorar a mortalidade hospitalar (motivo de internamento e de óbito) dos doentes com doença de Parkinson num hospital terciário, assim como as características demográficas e clínicas.

Material e Métodos: Identificação das admissões hospitalares dos doentes com o diagnóstico de doença de Parkinson entre 2008 e 2014 e seleção dos doentes falecidos. Revisão retrospetiva dos processos clínicos e inclusão dos doentes com doença confirmada clinicamente por um neurologista. Avaliação das causas de óbito, características demográficas e clínicas.

Resultados: Identificámos 1525 admissões hospitalares de doentes com diagnóstico de doença de Parkinson, das quais 150 resultaram em óbito. Destes, 52 cumpriam critérios de inclusão. A idade média do início dos sintomas de doença foi 66,8 anos ( $\pm 8,7$ ) e a duração média da doença foi 12,5 anos $( \pm 7,9)$. Sessenta e cinco por cento dos doentes encontravam-se no estádio 4-5 na escala de Hoehn e Yahr. Trinta e três doentes (63\%) apresentavam demência e onze depressão. As infeções foram a principal causa de morte (respiratória em $63 \%$ dos casos).

Discussão: À semelhança da literatura, a pneumonia foi a principal causa de morte hospitalar e a maioria dos doentes apresentava estádio avançado de doença e demência. Contrastando com outros estudos, não se verificou diminuição da esperança média de vida e as doenças cardiovasculares e o traumatismo não foram causas de morte na nossa população.

Conclusões: Apresentamos o primeiro estudo português de mortalidade na doença de Parkinson. A pneumonia é a principal causa de morte hospitalar. O estádio avançado de doença e a demência foram características comuns nestes doentes.

Palavras-chave: Doença de Parkinson/mortalidade; Hospitalização; Mortalidade Hospitalar; Portugal.

\section{ABSTRACT}

Introduction: Parkinson's disease is associated with high hospital mortality. Male gender, late age at onset, higher disability and the coexistence of cognitive impairment or depression have been suggested to be risk factors of death. Pneumonia and cardiovascular diseases are the leading causes of death.

Objective: To characterize the mortality (causes of hospital admission and death) of Parkinson's disease patients in a tertiary hospital, as well as demographic and clinical characteristics.

Material and Methods: Identification of hospital admissions of Parkinson's disease patients that resulted in inpatient death between 2008 and 2014. Retrospective review of medical files and inclusion of patients with disease clinically confirmed by a neurologist. Assessment of causes of death and demographic and clinical characteristics of patients

Results: 1525 hospital admissions of Parkinson's disease patients were identified, of which 150 resulted in death. Of these, 52 patients met the inclusion criteria. Mean age at onset of Parkinson's disease symptoms was 66.8 years $( \pm 8.7)$ and mean duration of disease was 12.5 years $( \pm 7.9)$. Sixty-five percent of patients were in stages $4-5$ of the Hoehn and Yahr scale. Thirty-three patients $(63 \%)$ had dementia and eleven (21\%) had depression. Infections were the leading cause of death (respiratory in $63 \%$ of cases).

Discussion: Similarly to literature, pneumonia was the leading cause of hospital death and most patients presented advanced disease stage and dementia. Contrarily to other studies, life expectancy was not reduced and cardiovascular diseases and trauma were not causes of death in our population.

Conclusions: This is the first Portuguese mortality study in Parkinson's disease. Pneumonia is the leading cause of death. Advanced disease stage and dementia were common features in these patients.

Keywords: Hospital Mortality; Hospitalization; Parkinson Disease/mortality; Portugal.

\section{INTRODUÇÃO}

A doença de Parkinson (DP) é uma patologia neurodegenerativa progressiva associada a elevada morbilidade, com impacto na utilização dos cuidados de saúde e na mortalidade.1,2 É uma das doenças crónicas mais comuns do sistema nervoso central e estima-se que a sua prevalência em Portugal seja de 1,29/1000. ${ }^{3}$ As taxas de mortalidade hospitalar na DP são aproximadamente 1,5 vezes superior às da população geral. ${ }^{4,5}$ Dados recentes sugerem que a mortalidade na DP aumentou ao longo do tempo, devendo-se possivelmente ao aumento significativo da esperança média de vida na população geral. ${ }^{6}$ Características como o sexo masculino, a idade avançada no início da doença, a duração da doença, o estádio avançado na escala de Hoehn e Yahr, a pontuação elevada na Unified Parkinson's Disease Rating Scale, a disfunção cognitiva e a depressão, têm vindo a ser apontadas como fatores de risco de

\footnotetext{
1. Serviço de Neurologia. Centro Hospitalar do Porto. Porto. Portugal.

$\triangle$ Autor correspondente: Joana Martins. jcsfmartins@gmail.com

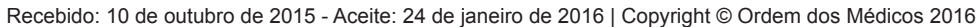


morte. ${ }^{1,7,8}$ As infeções respiratórias e as doenças cardiovasculares são as principais causas de morte na maioria dos estudos de mortalidade. ${ }^{7,9}$ Por outro lado, a mortalidade associada a doença oncológica ou vascular cerebral é menor nos doentes com DP, possivelmente pela menor prevalência destas doenças nestes doentes. ${ }^{6,10,11}$

Este estudo tem como objetivo principal explorar a mortalidade hospitalar (motivo de internamento e de óbito) dos doentes com DP num hospital terciário, assim como as suas características demográficas e clínicas.

\section{MATERIAL E MÉTODOS}

Para este estudo, identificámos as admissões hospitalares de doentes com o código 322 (DP) da 9a edição da Classificação Internacional de Doenças, no Centro Hospitalar do Porto, no período de tempo compreendido entre 1 de janeiro de 2008 e 31 de dezembro de 2014. Selecionámos os doentes que faleceram no contexto de urgência ou de internamento. Revimos os processos clínicos e incluímos no estudo os doentes com diagnóstico de DP realizado ou confirmado por um neurologista de acordo com os critérios de diagnóstico do Queen Square Brain Bank.12 Foram excluídos os doentes com diagnóstico clínico de síndrome parkinsónica atípica, parkinsonismo vascular, iatrogenia, doenças que cursam com alteração da marcha ou por falta de informação clínica. Recolhemos dados referentes às características demográficas e clínicas da DP (idade de início dos sintomas, estádio na escala de Hoehn e Yahr, demência e sintomas depressivos), motivo de hospitalização, duração do internamento e causas de óbito. A coexistência de demência foi inferida por queixas cognitivas descritas, pela toma de fármacos antidemenciais, ou, quando aplicável, através dos resultados de avaliação neuropsicológica

Tabela 1 - Características demográficas e clínicas dos doentes com DP falecidos em contexto hospitalar

\begin{tabular}{lc}
\hline Características clínicas & 52 \\
\hline Número de doentes, $\mathrm{n}$ & $28 / 24(54 / 46)$ \\
Sexo masculino/feminino, $\mathrm{n}(\%)$ & $79,7(6,9)$ \\
Idade média, anos (SD) & $66,8(8,7)$ \\
Idade média no início da doença, anos (SD) & $12,5(7,9)$ \\
Duração média de DP, anos (SD) & $79,7(6,9)$ \\
Idade média à data de óbito, anos (SD) & \\
Estádio na escala HY, n (\%) & $6(11,5)$ \\
1 - 2 & $10(19,2)$ \\
3 & $18(34,6)$ \\
4 & $16(30,8)$ \\
5 & $2(3,8)$ \\
Desconhecido & $33(63)$ \\
Demência, n (\%) & $11(21)$ \\
Depressão, n (\%) & \\
\hline
\end{tabular}

SD: desvio padrão; HY: escala de Hoehn e Yahr. formal (baseada na Dementia Rating Scale).

\section{RESULTADOS}

Entre janeiro de 2008 e dezembro de 2014 registaram-se 1525 admissões hospitalares de doentes com código de diagnóstico de DP. Identificaram-se 150 óbitos e destes, 52 (28 homens e 24 mulheres) cumpriam critérios de inclusão neste estudo. Destes doentes, quarenta eram seguidos numa consulta diferenciada em doenças do movimento (36 nesta instituição) e os restantes mantinham seguimento por um neurologista em regime particular.

A idade média do início dos sintomas de DP foi 66,8 anos $( \pm 8,7 ; \min =40$ e máx $=80)$ e a duração média da doença foi 12,5 anos $( \pm 7,9 ; \min =3$ e máx $=42)$. $A$ idade média à data de óbito foi 79,7 anos $( \pm 6,9$; $\min =62$ e máx =95). Seis doentes (12\%) apresentavam um estádio inferior ou igual a 2 na escala de Hoehn e Yahr, enquanto 46 (88\%) entre 3 e 5 . Trinta e três doentes (63\%) apresentam concomitantemente síndrome demencial e onze (21\%) estavam deprimidos (Tabela 1). Todos os doentes estavam medicados com levodopa (dose média $830 \mathrm{mg} / \mathrm{dia}$ ), três com agonista dopaminérgico (ropinirol de libertação prolongada $8 \mathrm{mg} / \mathrm{dia}$ ) e um com anticolinérgico (tri-hexifenidilo 5 $\mathrm{mg} / \mathrm{dia}$ ). Quanto a tratamento adjuvante, nove doentes estavam medicados com inibidor da COMT (entacapona 800 $\mathrm{mg} / \mathrm{dia}$ ), dois com inibidor da MAO-B (selegilina $5 \mathrm{mg} / \mathrm{dia}$ ) e dois com amantadina (400 mg/dia). Onze doentes estavam medicados com antipsicótico (quatro com clozapina, dose média $81 \mathrm{mg} /$ dia; quatro com quetiapina, dose média 194 $\mathrm{mg} / \mathrm{dia}$; e três com ciamemanzina $25 \mathrm{mg} / \mathrm{dia}$ ).

A duração média do internamento foi 10,8 dias $( \pm 14,2$; $\min =1$ e máx $=88$ ). Os principais motivos de hospitalização foram pneumonia em $67 \%$ dos doentes, sépsis com ponto de partida urinário em $12 \%$, acidente vascular cerebral em $8 \%$ e doença oncológica em fase terminal em $6 \%$.

A pneumonia foi a principal causa de morte, reportada em $63 \%$ dos doentes, seguida por sépsis de ponto de partida não-respiratório em $17 \%$, acidente vascular cerebral em $8 \%$ (dois enfartes cerebrais, uma hemorragia intracraniana espontânea e uma hemorragia subaracnoideia na sequência de rotura de aneurisma) e cancro em $8 \%$ (carcinomatose peritoneal, linfoma anaplásico da face, neoplasia do cárdia e tumor do pulmão de pequenas células). Isquemia mesentérica e tromboembolismo pulmonar foram causas menos comuns (em 2\%) (Tabela 2).

Tabela 2 - Causas de morte dos doentes com DP hospitalizados

\begin{tabular}{lc}
\hline Causas de morte & $\mathbf{n}(\%)$ \\
\hline Pneumonia & $33(63)$ \\
Infeção não-respiratória & $9(17)$ \\
Acidente vascular cerebral & $4(8)$ \\
Cancro & $4(8)$ \\
Tromboembolismo pulmonar & $1(2)$ \\
Isquemia mesentérica & $1(2)$ \\
\hline
\end{tabular}




\section{DISCUSSÃO}

Tem sido afirmado que os doentes com DP morrem mais precocemente do que a população geral. No entanto, na nossa série constatou-se que a idade média à data do óbito (79,7 anos) aproxima-se da esperança média de vida dos portugueses, segundo os dados oficiais do Instituto $\mathrm{Na}$ cional de Estatística correspondentes a 2011-2013 (80,13 anos). ${ }^{13}$ Verificou-se também que o tempo médio entre o diagnóstico e a morte é relativamente longo (12,5 anos) e que $65 \%$ dos doentes se encontravam num estádio avançado da doença (4-5 na escala de Hoehn e Yahr), levantando a questão da qualidade de vida e da importância da preservação da funcionalidade e da independência do doente. Constatou-se que os doentes em fases mais precoces de $\mathrm{DP}$ (i.e. estádio HY $\leq 2$; seis doentes com idade média de 76 anos e duração média de doença de quatro anos) faleceram por pneumonia ou por acidente vascular cerebral, de forma equitativa. Consideramos provável a contribuição de comorbilidades e fatores de risco comuns nesta faixa etária em ambos os casos.

A pneumonia foi a principal causa de morte, achado que é consistente com diferentes estudos de mortalidade na DP. ${ }^{7,9,10}$ Este facto poderá advir da rigidez da parede torácica durante as flutuações motoras, das discinésias e da disfunção das vias aéreas superiores, às quais se associa um risco aumentado de aspiração e uma menor eficácia da expetoração. ${ }^{14}$ Intervenções como mobilização frequente dos doentes e cinesioterapia podem diminuir o risco de intercorrências infeciosas e, consequentemente, de morte. Neste estudo não se verificou nenhum óbito decorrente de doença cardíaca isquémica, contrastando com vários autores que apontam as doenças cardiovasculares como uma das principais causas de morte dos doentes com DP.., 10 Considerando os efeitos cardiovasculares nocivos dos fármacos antipsicóticos, especulamos a existência de uma relação entre a ausência de morte de causa cardiovascular e uma menor utilização deste tipo de fármaco neste grupo de doentes. Notamos que apenas $20 \%$ dos doentes estavam medicados com doses baixas de antipsicóticos. A doença vascular cerebral e o cancro, que em outras séries são causas incomuns de morte, no nosso coorte foram responsáveis por $16 \%$ dos óbitos, o que poderá estar relacionado com a idade média mais avançada dos nossos doentes. Apesar das elevadas taxas de hospitalização por traumatismo na DP, particularmente decorrentes de quedas, na nossa série não se verificou nenhuma morte secundária a trauma. ${ }^{14}$

A demência tem sido considerada por alguns autores como um fator de risco de morte neste grupo, o que pode resultar do impacto da disfunção cognitiva no estado funcional destes doentes. ${ }^{1,4,8}$ Quase dois terços dos nossos doentes apresentavam síndrome demencial, o que corresponde a uma prevalência superior ao descrito em algumas séries recentes. Oosterveld et al descrevem a presença de demência em $35 \%$ dos doentes, enquanto que Lau et al descrevem uma prevalência de $31 \% .{ }^{1,8}$ À semelhança de outros estudos, nesta série a depressão é pouco frequente, mas tem sido demonstrado que esta é preditiva de morte precoce quando presente..$^{15}$ Intervenções precoces no tratamento das comorbilidades associadas ao aumento da mortalidade, como a demência ou a depressão, podem melhorar o prognóstico destes doentes.

As principais vantagens deste estudo resultam da inclusão de doentes com DP confirmada e com seguimento por neurologista e da existência de um registo hospitalar das circunstâncias da morte. As principais limitações estão inerentes à sua natureza retrospetiva e ao facto de ser um estudo monocêntrico.

\section{CONCLUSÃO}

O presente estudo é o primeiro estudo português de mortalidade na DP. Apresentamos resultados concordantes com outros estudos de mortalidade publicados na literatura, relativamente ao estádio avançado de doença e à coexistência de demência como características comuns destes doentes. As doenças infeciosas são a principal causa de morte hospitalar na DP, com um claro predomínio da infeção pulmonar.

\section{PROTECÇÃO DE PESSOAS E ANIMAIS}

Os autores declaram que os procedimentos seguidos estavam de acordo com os regulamentos estabelecidos pelos responsáveis da Comissão de Investigação Clínica e Ética e de acordo com a Declaração de Helsínquia da Associação Médica Mundial.

\section{CONFIDENCIALIDADE DOS DADOS}

Os autores declaram ter seguido os protocolos do seu centro de trabalho acerca da publicação dos dados de doentes.

\section{CONFLITO DE INTERESSES}

Os autores declaram não ter nenhum conflito de interesses relativamente ao presente artigo.

\section{FONTES DE FINANCIAMENTO}

Não existiram fontes externas de financiamento para a realização deste artigo.

\section{REFERÊNCIAS}

1. Oosterveld LP, Allen JC, Reinoso G, Seah S-H, Tay K-Y, Au W-L, et al. Prognostic factors for early mortality in Parkinson's disease. Parkinsonism Relat Disord. 2015;21:226-30.

2. Chou KL, Zamudio J, Schmidt P, Price CC, Parashos SA, Bloem BR, et al. Hospitalization in Parkinson disease: A survey of National Parkinson Foundation Centers. Parkinsonism Relat Disord. 2011;17:440-5.

3. Ferreira JJ, Gonçalves N, Valadas A, Januário C, Silva MR, Nogueira L, et al. Estudo epidemiológico de avaliação da prevalência da doença de Parkinson em Portugal. Sinapse. 2014;14:66.

4. Diem-Zangerl A, Seppi K, Wenning GK, Trinka E, Ransmayr G, Oberaigner W, et al. Mortality in Parkinson's disease: A 20-year followup study. Mov Disord. 2009;24:819-25. 
5. Hoehn MM, Yahr MD. Parkinsonism: onset, progression and mortality. Neurology. 1967;17:427-42.

6. Macleod AD, Taylor KSM, Counsell CE. Mortality in Parkinson's disease: A systematic review and meta-analysis. Mov Disord. 2014;29:1615-22.

7. Xu J, Gong DD, Man CF, Fan Y. Parkinson's disease and risk of mortality: meta-analysis and systematic review. Acta Neurol Scand. 2014;129:719.

8. de Lau LM, Verbaan D, Marinus J, van Hilten JJ. Survival in Parkinson's disease. Relation with motor and non-motor features. Parkinsonism Relat Disord. 2014;20:613-6.

9. Pinter B, Diem-Zangerl A, Wenning GK, Scherfler C, Oberaigner W, Seppi K, et al. Mortality in Parkinson's disease: a 38-year follow-up study. Mov Disord. 2015;30:266-9.

10. Pennington $S$, Snell K, Lee M, Walker R. The cause of death in idiopathic Parkinson's disease. Parkinsonism Relat Disord. 2010;16:434-7.
11. Fall PA, Saleh A, Fredrickson M, Olsson JE, Granérus AK. Survival time, mortality, and cause of death in elderly patients with Parkinson's disease: a 9-year follow-up. Mov Disord. 2003;18:1312-6.

12. Hughes AJ, Daniel SE, Kilford L, Lees AJ. Accuracy of clinical diagnosis of idiopathic Parkinson's disease: a clinico-pathological study of 100 cases. J Neurol Neurosurg Psychiatry. 1992;55:181-4.

13. Ishihara LS, Cheesbrough A, Brayne C, Schrag A. Estimated life expectancy of Parkinson's patients compared with the UK population. J Neurol Neurosurg Psychiatry. 2007;78:1304-9.

14. Martignoni E, Godi L, Citterio A, Zangaglia R, Riboldazzi G, Calandrella $\mathrm{D}$, et al. Comorbid disorders and hospitalisation in Parkinson's disease: a prospective study. Neurol Sci. 2004;25:66-71.

15. Hughes TA, Ross HF, Mindham RHS, Spokes EG. Mortality in Parkinson's disease and its association with dementia and depression. Acta Neurol Scand. 2004;110:118-23. 